\title{
Transient Dynamics in Magnetic Force Microscopy for a Single-Spin Measurement
}

\author{
G.P. Berman, ${ }^{1}$ F. Borgonovi, ${ }^{2}$ G. V. López, ${ }^{3}$ V.I. Tsifrinovich ${ }^{4}$ \\ ${ }^{1}$ Theoretical Division and CNLS, Los Alamos National Laboratory, Los \\ Alamos, NM 87545 \\ ${ }^{2}$ Dipartimento di Matematica e Fisica, Università Cattolica, via Musei 41 , \\ 25121 Brescia, Italy, and I.N.F.M., Unità di Brescia and I.N.F.N., sezione \\ di Pavia \\ ${ }^{3}$ Departamento de Física de la Universidad de Guadalajara S.R. 500, 44420 \\ Guadalajara, Jalisco, México \\ ${ }^{4}$ IDS Department, Polytechnic University, Six Metrotech Center, Brooklyn, \\ New York 11201
}

\begin{abstract}
We analyze a single-spin measurement using a transient process in magnetic force microscopy (MFM) which could increase the maximum operating temperature by a factor of $Q$ (the quality factor of the cantilever) in comparison with the static Stern-Gerlach effect. We obtain an exact solution of the master equation, which confirms this result. We also discuss the conditions required to create a macroscopic Schrödinger cat state in the cantilever.
\end{abstract}

\section{Introduction}

The routine magnetic force microscopy (MFM) seems to be of a little use for a single spin detection in solids. Even for unrealistically small distances between the ferromagnetic particle on the cantilever tip and the spin, the maximum temperature for a single-spin measurement comes to a millikelvin region [1]. In this paper, we show that using a transient process, one can increase the maximum temperature of a single-spin measurement by a factor of $Q$ (the quality factor of the cantilever). Alternatively, one can increase the distance between the ferromagnetic particle and the cantilever tip if one is willing to work at millikelvin temperatures. 
In section 2 we explain the basic idea of our work. In sections 3-5 we obtain an exact solution of the master equations, which confirms our idea. In section 6 we derive the conditions for creating a macroscopic Schrödinger cat state (MSCS) in MFM. In Conclusion we summarize our results.

\section{Transient Process in MFM}

In this section we describe the basic idea of our work. Suppose that a ferromagnetic particle on the cantilever tip interacts with a single spin on the solid surface. (See Fig. 1.) The equilibrium position of the cantilever tip depends on the spin direction. The distance between two possible equilibrium positions, corresponding to two spin stationary states, is given by $2 F / k_{c}$, where $k_{c}$ is the cantilever spring constant, $F$ is the magneto-static force between the ferromagnetic particle and the spin. In order to measure the state of the spin, this distance must be greater than twice the uncertainty due to the thermodynamical noise of the cantilever position. This uncertainty can be estimated as $\left(k_{B} T / k_{c}\right)^{1 / 2}$, where $k_{B}$ is the Boltzmann's constant and $T$ is the temperature. Thus, the condition for a single spin measurement (the static Stern-Gerlach effect) is [1]

$$
T<T_{\max }=F^{2} / k_{B} k_{c}
$$

Now, assume that we quickly change the stationary state of the spin and consider the transient cantilever vibrations after this change. The amplitude of the cantilever vibrations at time $t \ll Q / \omega_{c}\left(\omega_{c}\right.$ is the cantilever frequency and $Q / \omega_{c}$ is the time constant of the cantilever) is $4 F / k_{c}$. In addition, assume that we detect the position and momentum of a point on the cantilever tip with an accuracy that satisfies the quantum limit $\left(\delta P_{Z}\right)(\delta Z) \approx \hbar / 2$ (the cantilever oscillates along the z-axis.) To find out the state of the spin we are going to compare the observed trajectory of the cantilever tip with the theoretical prediction. The theory predicts the cantilever trajectory within an uncertainty due to the thermal noise. However, the thermal uncertainty of the cantilever position at time $t \ll Q / \omega_{c}$ increases as $\left[t\left(\omega_{c} / Q\right)\left(k_{B} T / k_{c}\right)\right]^{1 / 2}$. We can obtain this expression assuming an initial $\left(t \ll Q / \omega_{c}\right)$ thermal dif-

fusion with two common properties: 1) The dispersion $\overline{(\delta Z)^{2}}$ is proportional 
to time, $t$, and 2) The uncertainty of the cantilever position equals its thermodynamical value if we formally put $t=Q / \omega_{c}$.

At the time $t=\pi / \omega_{c}$ (half of the first period), the distance between two possible cantilever positions takes its maximum possible value $4 F / k_{c}$. At the same time, the thermal uncertainty of the position predicted by the theory is still much smaller than its thermodynamical value. Now, the condition for a single spin measurement is

$$
T<T_{\max }=\frac{4 Q F^{2}}{\pi k_{B} k_{c}} .
$$

One can see that the maximum temperature for a single spin measurement increases by a factor of $Q$, compared with the static Stern-Gerlach effect.

In the next three sections, we will confirm this estimation by direct solution of the master equation.

\section{The Hamiltonian and the Master Equation}

We assume that the cantilever tip with an attached ferromagnetic particle can oscillate along the z-axis. (See Fig. 1.) A single paramagnetic atom with spin $1 / 2$ is placed near the cantilever tip. The dimensionless Hamiltonian of the cantilever tip interacting with a single spin is

$$
\hat{H}=\frac{1}{2}\left(\hat{p}_{z}^{2}+\hat{z}^{2}\right)-2 \eta \hat{z} \hat{S}_{z}
$$

We introduced the following notation (below we omit hats for operators):

$$
z=Z / Z_{q}, \quad p_{z}=P_{z} / P_{q}, \quad \eta=g \mu_{B}\left|\frac{\partial B_{z}}{\partial z}\right| / 2 F_{q},
$$

where $Z_{q}, P_{q}$ and $F_{q}$ are the "quants" of the coordinate, momentum and force acting on the cantilever,

$$
Z_{q}=\left(\frac{\hbar \omega_{c}}{k_{c}}\right)^{1 / 2}, \quad P_{q}=\hbar / Z_{q}, \quad F_{q}=k_{c} Z_{q}
$$


The variables $Z$ and $P_{z}$ are the "dimensional" coordinate and momentum of the cantilever tip, $k_{c}$ is the cantilever "spring constant", $\omega_{c}$ is its frequency, $g$ is the "g-factor" of the spin (below we use $g=2$ ), $\partial B_{z} / \partial z$ is the magnetic field gradient produced by the ferromagnetic particle at the spin location when the cantilever is in the equilibrium position with no spin $(z=0)$. Note that the cantilever interacts with the z-component of the spin, which is an integral of motion in our system. In the Hamiltonian (3) we omitted the term $\left(g \mu_{B} B_{0} / \hbar \omega_{c}\right) \hat{S}_{z}$, where $B_{0}$ is the magnetic field on the spin when the cantilever is at the origin $(z=0)$. This term may be eliminated "physically" (by application of a uniform external field of magnitude $B_{0}$ in the negative $z$ direction) or "mathematically" (by transferring to the system of coordinates rotating with the frequency $\left.g \mu_{B} B_{0} / \hbar\right)$.

The master equation describes the evolution of the density matrix of the system interacting with the environment (see for example [2-5]). We are taking into account the interaction of the cantilever with its environment, and ignore the direct interaction between the spin and the environment, assuming that the spin relaxation and decoherence times are large enough. The effect of the environment depends on its "spectral density", i.e. the density of environmental oscillators at a given frequency. Probably, the simplest model of the environment is the "ohmic" model, where the spectral density is proportional to the frequency $\omega$ for $\omega<\Omega$, where $\Omega$ is the cutoff frequency for the environment. A master equation for the non-ohmic environment has been derived in [5]. For the ohmic model, the simplest master equation has been obtained in [2]. This equation is valid in the "high temperature limit" $k_{B} T>>\hbar \Omega$. The master equation derived in [3] is valid for arbitrary temperature. As pointed out in [5], both equations [2] and [3] fail at times shorter than or close to $\hbar / k_{B} T$.

We are going to consider the "gedanken experiment" discussed in [1]. Suppose that initially $(t=0)$ the spin is in a superposition of the two states with the $z$-projection of the spin $S_{z}=1 / 2$ and $S_{z}=-1 / 2$. These two states of the spin correspond to two different equilibrium positions of the cantilever tip. Thus, the cantilever (without decoherence) would transform into a MSCS: two simultaneous equilibrium positions. Certainly, decoherence will destroy this state. The master equation describes both the appearance of the MSCS and its destruction due to decoherence. 
Following [1], we consider the ultra-thin cantilever reported on [6]. It has the spring constant $k_{c}=6.5 \times 10^{-6} \mathrm{~N} / \mathrm{m}$, the frequency, $\omega_{c} / 2 \pi=1.7 \mathrm{kHz}$, and the quality factor, $Q=6700$. The ferromagnetic particle on the cantilever tip is taken to be a sphere of radius $R=15 \mathrm{~nm}$ at a distance $5 \mathrm{~nm}$ from the paramagnetic atom. (Below we consider conditions for increasing the distance between the cantilever and spin.) For this case, the static displacement of the cantilever tip due to its interaction with the single spin exceeds the thermal vibrations of the cantilever for temperatures

$$
T<T_{\max }=\frac{\left(\mu_{B} \partial B_{z} / \partial z\right)^{2}}{k_{B} k_{c}} \approx 1.7 \mathrm{mK} .
$$

In our gedanken experiment for the temperature $T>>\hbar \omega_{c} / k_{B} \approx 8 \times 10^{-8} \mathrm{~K}$, we can use the simplest high temperature limit in the "ohmic model".

The master equation in the high temperature limit can be written in the form [2]

$$
\begin{aligned}
\frac{\partial \rho_{s, s^{\prime}}}{\partial \tau} & =\left[\frac{i}{2}\left(\partial_{z z}-\partial_{z^{\prime} z^{\prime}}\right)-\frac{i}{2}\left(z^{2}-z^{\prime 2}\right)-\frac{1}{2} \beta\left(z-z^{\prime}\right)\left(\partial_{z}-\partial_{z^{\prime}}\right)-D \beta\left(z-z^{\prime}\right)^{2}\right. \\
& \left.-2 i \eta\left(z^{\prime} s^{\prime}-z s\right)\right] \rho_{s, s^{\prime}}
\end{aligned}
$$

Here, $s$ and $s^{\prime}$ take values $\pm 1 / 2$ (we use $s$ instead of $S_{z}$ ), $\tau=\omega_{c} t, \beta=1 / Q$ and $D=k_{B} T / \hbar \omega_{c}$. Using new coordinates

$$
r=z-z^{\prime}, \quad R=\frac{1}{2}\left(z+z^{\prime}\right) .
$$

Eq. (7) can be written as:

$$
\begin{aligned}
& \frac{\partial \rho_{s, s^{\prime}}(R, r, \tau)}{\partial \tau}= \\
& \left\{i \partial_{R r}-i R r-\beta r \partial_{r}-D \beta r^{2}-i \eta\left[(2 R-r) s^{\prime}-(2 R+r) s\right]\right\} \rho_{s, s^{\prime}}(R, r, \tau) .
\end{aligned}
$$


Performing a Fourier transformation of this equation with respect to the variable "R", one obtains, after re-arrangements,

$$
\frac{\partial \hat{\rho}_{s, s^{\prime}}}{\partial \tau}+(\beta r-k) \frac{\partial \hat{\rho}_{s, s^{\prime}}}{\partial r}+\left[r+2 \eta\left(s^{\prime}-s\right)\right] \frac{\partial \hat{\rho}_{s, s^{\prime}}}{\partial k}=\left[-D \beta r^{2}+i \eta r\left(s^{\prime}+s\right)\right] \hat{\rho}_{s, s^{\prime}},
$$

where

$$
\hat{\rho}_{s, s^{\prime}}(k, r, \tau)=\int_{-\infty}^{+\infty} e^{i k R} \rho_{s, s^{\prime}}(R, r, \tau) d R
$$

We can study separately the spin diagonal case $\left(s=s^{\prime}\right)$ and the off-diagonal case $\left(s \neq s^{\prime}\right)$. For $s^{\prime}=s$ (up-up or down-down spins), we have the following equation:

$$
\frac{\partial \hat{\rho}_{s, s}}{\partial \tau}+(\beta r-k) \frac{\partial \hat{\rho}_{s, s}}{\partial r}+r \frac{\partial \hat{\rho}_{s, s}}{\partial k}=\left(-D \beta r^{2}+2 i \eta r s\right) \hat{\rho}_{s, s},
$$

and for $s^{\prime} \neq s$ (up-down or down-up spins):

$$
\frac{\partial \hat{\rho}_{s,-s}}{\partial \tau}+(\beta r-k) \frac{\partial \hat{\rho}_{s,-s}}{\partial r}+(r+4 \eta s) \frac{\partial \hat{\rho}_{s,-s}}{\partial k}=-D \beta r^{2} \hat{\rho}_{s,-s} .
$$

We will derive the exact solution of the master equation (7) for the case when the spin is "prepared" initially in the superposition of two states with $s=1 / 2$ and $s=-1 / 2$, while the cantilever tip is in the quasiclassical coherent state

$$
\psi(z, s, 0)=\frac{1}{(\pi)^{1 / 4}} \exp \left[i p_{0} z-\left(z-z_{0}\right)^{2} / 2\right] \otimes\left(\begin{array}{l}
a \\
b
\end{array}\right)
$$

where the amplitudes $a$ and $b$ correspond to the values of $s=1 / 2$ and $s=-1 / 2$ respectively. The corresponding density matrix can be written as

$$
\rho_{s s^{\prime}}\left(z, z^{\prime}, 0\right)=\psi(z, s, 0) \otimes \psi^{\dagger}\left(z^{\prime}, s^{\prime}, 0\right) .
$$

Note that we consider an ensemble of spin-cantilever systems with the same initial state. This implies that the experimenter can detect the position and momentum of a point on the cantilever tip with quantum limit accuracy $\overline{\left(\delta p_{z}\right)^{2}} \overline{(\delta z)^{2}}=1 / 4$. (In our gedanken experiment, this corresponds to an uncertainty of $300 \mathrm{fm}$ for position and $300 \mathrm{~nm} / \mathrm{s}$ for velocity.) Based on the master equation, we can predict the average position of the cantilever tip for 
its given initial state, depending on the spin state. If the double uncertainty of the position is smaller than the separation between two possible average positions, the cantilever tip will measure the state of the spin.

After Fourier transformation, the "cantilever part" of the density matrix is represented by

$$
\hat{\rho}_{s, s^{\prime}}(k, r, 0) \propto \exp \left[i p_{0} r+i k z_{0}-r^{2} / 4-k^{2} / 4\right] .
$$

\section{Solution for spin diagonal matrix elements}

The equations for the characteristics of Eq. (11) are

$$
d \tau=\frac{d r}{\beta r-k}=\frac{d k}{r}=\frac{d \hat{\rho}_{s, s^{\prime}}}{\left(-D \beta r^{2}+2 i \eta s r\right) \hat{\rho}_{s s}},
$$

or, explicitly

$$
\begin{aligned}
& \frac{d r}{d \tau} \quad=\beta r-k \\
& \frac{d k}{d \tau} \quad=r \\
& \frac{d \hat{\rho}_{s, s^{\prime}}}{d \tau}=\left(-D \beta r^{2}+2 i \eta s r\right) \hat{\rho}_{s, s^{\prime}}
\end{aligned}
$$

From the first two equations in (17), one obtains

$$
\frac{d^{2} k}{d \tau^{2}}-\beta \frac{d k}{d \tau}+k=0
$$

which has the following general solution

$$
k=e^{\beta \tau / 2}\left(c_{1} \cos \theta \tau+c_{2} \sin \theta \tau\right),
$$

where $\theta=\sqrt{1-\frac{\beta^{2}}{4}}$. (Here we are considering the case $\beta<2$ so $\theta$ is a real number. The case $\beta>2$ can also be solved analytically.) Using the second equation in (17) one obtains:

$$
r=e^{\beta \tau / 2}\left[\left(\frac{\beta}{2} \cos \theta \tau-\theta \sin \theta \tau\right) c_{1}+\left(\frac{\beta}{2} \sin \theta \tau+\theta \cos \theta \tau\right) c_{2}\right] .
$$


Inverting Eqs. (18) and (19) as a function of $c_{1}$ and $c_{2}$ one obtain the characteristic curves:

$$
c_{1}=e^{-\beta \tau / 2}\left(q_{1} k+q_{2} r\right)
$$

and

$$
c_{2}=e^{-\beta \tau / 2}\left(p_{1} k+p_{2} r\right),
$$

where the time dependent constants $q_{1}, q_{2}, p_{1}$ and $p_{2}$ have been defined as

$$
\begin{aligned}
& q_{1}=\frac{1}{\theta}\left(\frac{\beta}{2} \sin \theta \tau+\theta \cos \theta \tau\right), \\
& q_{2}=-\frac{1}{\theta} \sin \theta \tau, \\
& p_{1}=\frac{1}{\theta}\left(-\frac{\beta}{2} \cos \theta \tau+\theta \sin \theta \tau\right), \\
& p_{2}=\frac{1}{\theta} \cos \theta \tau .
\end{aligned}
$$

Substituting (19) into the third equation of (17) and integrating in time, one obtains:

$$
\hat{\rho}_{s, s}(k, r, \tau) \propto Q\left(c_{1}, c_{2}\right) \exp \left[i 2 \eta s\left(c_{1} g_{1}+c_{2} g_{2}\right)-D \beta\left(c_{1}^{2} f_{1}+2 c_{1} c_{2} f_{3}+c_{2}^{2} f_{2}\right)\right]
$$

where the functions $f_{i}^{\prime} s$ and $g_{i}^{\prime} s$ are defined as

$$
\begin{aligned}
& f_{1}(\tau)=\frac{e^{\beta \tau}}{8}\left[\left(\beta+\frac{4 \theta^{2}}{\beta}\right)+\beta \cos 2 \theta \tau-2 \theta \sin 2 \theta \tau\right], \\
& f_{2}(\tau)=\frac{e^{\beta \tau}}{8}\left[\left(\beta+\frac{4 \theta^{2}}{\beta}\right)-\beta \cos 2 \theta \tau+2 \theta \sin 2 \theta \tau\right], \\
& f_{3}(\tau)=\frac{e^{\beta \tau}}{8}[2 \theta \cos 2 \theta \tau+\beta \sin 2 \theta \tau], \\
& g_{1}(\tau)=e^{\beta \tau / 2} \cos \theta \tau, \\
& g_{2}(\tau)=e^{\beta \tau / 2} \sin \theta \tau .
\end{aligned}
$$


The arbitrary function $A$ which depends on the characteristics is determined by the initial density matrix $\hat{\rho}_{s, s}(k(0), r(0), 0)$,

$$
\begin{aligned}
A\left(c_{1}, c_{2}\right)= & \hat{\rho}_{s, s}\left(c_{1}, \frac{1}{2} \beta c_{1}+\theta c_{2}, 0\right) \exp \left[-2 i \eta s\left(c_{1} g_{10}+c_{2} g_{20}\right)\right] \\
& \times \exp \left[D \beta\left(c_{1}^{2} f_{10}+2 c_{1} c_{2} f_{30}+c_{2}^{2} f_{20}\right)\right],
\end{aligned}
$$

where $f_{i 0}=f_{i}(0)$ and $g_{i 0}=g_{i}(0)$. From the initial density matrix (Eq. (15)), we obtain

$$
\begin{aligned}
\hat{\rho}_{s, s^{\prime}}(k, r, 0) & \propto \exp \left\{i\left[\left(\frac{1}{2} p_{0} \beta+z_{0}\right) c_{1}+p_{0} \theta c_{2}\right]\right\} \\
& \times \exp \left\{-\left[\left(\frac{\beta^{2}}{16}+\frac{1}{4}\right) c_{1}^{2}+\frac{\beta \theta}{4} c_{1} c_{2}+\frac{\theta^{2}}{4} c_{2}^{2}\right]\right\} .
\end{aligned}
$$

Substituting (25) and (26) into (23) one obtains:

$$
\begin{aligned}
\hat{\rho}_{s, s}(k, r, \tau) \propto & \exp \left\{i\left[\left(\frac{1}{2} p_{0} \beta+z_{0}+2 \eta s G_{1}\right) c_{1}+\left(p_{0} \theta+2 \eta s G_{2}\right) c_{2}\right]\right\} \\
& \times \exp \left\{-\left[\left(\frac{\beta^{2}}{16}+\frac{1}{4}\right) c_{1}^{2}+\frac{\beta \theta}{4} c_{1} c_{2}+\frac{\theta^{2}}{4} c_{2}^{2}\right]\right\} \\
& \times \exp \left\{-D \beta\left(F_{1} c_{1}^{2}+2 c_{1} c_{2} F_{3}+F_{2} c_{2}^{2}\right)\right\},
\end{aligned}
$$

where $F_{i}$ and $G_{i}$ are defined as

$$
F_{i}(\tau)=f_{i}(\tau)-f_{i 0}, \quad G_{i}(\tau)=g_{i}(\tau)-g_{i 0} .
$$

Substituting in (27) the values of characteristics as a function of $k$ and $r$ (Eqs.(20) and (21)), one obtains:

$$
\hat{\rho}_{s s}(k, r, \tau) \propto \exp \left[-r^{2} C_{1}+i r C_{2}+\left(i B_{2}-r B_{1}\right) k-\sigma_{*}^{2} k^{2}\right],
$$

where

$$
\sigma_{*}^{2}=e^{-\beta t}\left[\left(\frac{\beta^{2}}{16}+\frac{1}{4}\right) q_{1}^{2}+\frac{\beta \theta}{4} q_{1} p_{1}+\frac{\theta^{2}}{4} p_{1}^{2} \quad+D \beta\left(F_{1} q_{1}^{2}+2 q_{1} p_{1} F_{3}+F_{2} p_{1}^{2}\right)\right],
$$




$$
\begin{gathered}
B_{1}=e^{-\beta t}\left\{\left(\frac{\beta^{2}}{16}+\frac{1}{4}\right) 2 q_{1} q_{2}+\frac{\beta \theta}{4}\left(q_{1} p_{2}+q_{2} p_{1}\right)+\frac{\theta^{2}}{4} 2 p_{1} p_{2}\right. \\
\left.+2 D \beta\left[F_{1} q_{1} q_{2}+\left(q_{1} p_{2}+q_{2} p_{1}\right) F_{3}+F_{2} p_{1} p_{2}\right]\right\} \\
B_{2}(s)=e^{-\beta t / 2}\left[\left(\frac{1}{2} p_{0} \beta+z_{0}+2 \eta s G_{1}\right) q_{1}+\left(p_{0} \theta+2 \eta s G_{2}\right) p_{1}\right], \\
C_{1}=e^{-\beta t}\left[\left(\frac{\beta^{2}}{16}+\frac{1}{4}\right) q_{2}^{2}+\frac{\beta \theta}{4} q_{2} p_{2}+\frac{\theta^{2}}{4} p_{2}^{2}\right. \\
\left.+D \beta\left(F_{1} q_{2}^{2}+2 q_{2} p_{2} F_{3}+F_{2} p_{2}^{2}\right)\right] \\
C_{2}(s)=e^{-\beta t / 2}\left[\left(\frac{1}{2} p_{0} \beta+z_{0}+2 \eta s G_{1}\right) q_{2}+\left(p_{0} \theta+2 \eta s G_{2}\right) p_{2}\right] .
\end{gathered}
$$

In Eqs. (31) and (33) the explicit dependence on $s$ is presented. Performing the inverse Fourier transform one obtains

$$
\begin{aligned}
& \rho_{1 / 2,1 / 2}(R, r, \tau)= \frac{|a|^{2}}{\sqrt{\pi} \sigma_{*}} \exp \left[-r^{2} C_{1}+i r C_{2}(1 / 2)\right] \\
& \times \exp \left[\left(-r B_{1}+i B_{2}(1 / 2)-i R\right)^{2} / 4 \sigma_{*}^{2}\right], \\
& \rho_{-1 / 2,-1 / 2}(R, r, \tau)=\frac{|b|^{2}}{\sqrt{\pi} \sigma_{*}} \exp \left[-r^{2} C_{1}+i r C_{2}(-1 / 2)\right] \\
& \times \exp \left[\left(-r B_{1}+i B_{2}(-1 / 2)-i R\right)^{2} / 4 \sigma_{*}^{2}\right] .
\end{aligned}
$$

Eqs. (34) represent two squeezed Gaussians with modulus

$$
\begin{aligned}
&\left|\rho_{1 / 2,1 / 2}(R, r, \tau)\right|=\frac{|a|^{2}}{\sqrt{\pi} \sigma_{*}} \exp \left[-r^{2}\left(C_{1}-B_{1}^{2} / 4 \sigma_{*}^{2}\right)\right] \\
& \times \exp \left[-\left(B_{2}(1 / 2)-R\right)^{2} / 4 \sigma_{*}^{2}\right], \\
&\left|\rho_{-1 / 2,-1 / 2}(R, r, \tau)\right|=\frac{|b|^{2}}{\sqrt{\pi} \sigma_{*}} \exp \left[-r^{2}\left(C_{1}-B_{1}^{2} / 4 \sigma_{*}^{2}\right)\right] \\
& \times \exp \left[-\left(B_{2}(-1 / 2)-R\right)^{2} / 4 \sigma_{*}^{2}\right] .
\end{aligned}
$$


Fig. 2 shows schematically two peaks (seen from the top as ellipses) corresponding to the two matrix elements $\left|\rho_{1 / 2,1 / 2}\right|$ and $\left|\rho_{-1 / 2,-1 / 2}\right|$. We denote the centers of the ellipses, which lie on the diagonal $z=z^{\prime}$, by $M_{++}$and $M_{--}$, the semi-major axis by $\sigma_{d}$, the semi-minor axis by $\sigma_{d}^{\prime}$ and the distance between the centers by $\Delta_{d}$. The $\rho_{1 / 2,1 / 2}^{\max }$ is located at $M_{++}=\left(r=0, R=B_{2}(1 / 2)\right)$ or $z=z^{\prime}=B_{2}(1 / 2)$, while the $\rho_{-1 / 2,-1 / 2}^{\max }$ is at $M_{--}=\left(r=0, R=B_{2}(-1 / 2)\right)$ or $\left(z=z^{\prime}=B_{2}(-1 / 2)\right)$. The distance $\Delta_{d}$ is given by

$$
\Delta_{d}=B_{2}(1 / 2)-B_{2}(-1 / 2) .
$$

From Eqs. (35), we obtain $\sigma_{d}=\sqrt{2} \sigma_{*}$, and

$$
2{\sigma^{\prime}}_{d}^{2}=\frac{4 \sigma_{*}^{2}}{4 \sigma_{*}^{2} C_{1}-B_{1}^{2}}
$$

For a single spin measurement, the two peaks corresponding to $\rho_{1 / 2,1 / 2}^{\max }$ and $\rho_{-1 / 2,-1 / 2}^{\max }$ must be well separated. It follows that the condition $\Delta_{d}>2 \sigma_{d}$ must be satisfied.

First, we consider the case $\beta \tau \gg 1$ or $\tau \gg Q / \omega_{c}$, where $Q / \omega_{c}$ is the time constant for the cantilever. In this case, we obtain two equilibrium positions for the cantilever, when the transient process is over. We have $\Delta_{d}=2 \eta$ and $\sigma_{d}=\sqrt{D}$. The value $\sigma_{d}=\sqrt{D}$ is the thermodynamical uncertainty in the cantilever position caused by the thermal noise. The two equilibrium positions can be distinguished if $\eta>\sqrt{D}$ or $T<F^{2} / k_{B} k_{c}$, where $F=\mu_{B}\left|\partial B_{z} / \partial z\right|$ is the magneto-static force between the ferromagnetic particle and the paramagnetic atom. The last expression exactly coincides with formula (1).

Next, we consider the initial transient process after the instant $(t=0)$ at which the paramagnetic spin has been transferred into the superpositional state. For $\beta \tau \ll 1$, we have

$$
\Delta_{d}=4 \eta \sin ^{2} \frac{\tau}{2}, \quad \sigma_{d}=\left[1 / 2+D \beta \tau-D \beta \cos \tau \sin ^{3} \tau\right]^{1 / 2} .
$$

This expression for $\Delta_{d}$ describes the oscillating distance between the two peaks. It corresponds to initial vibrations of two classical oscillators near their equilibrium positions $z=\eta$ and $z=-\eta$. The distance between them 
is given by $\Delta_{d}$. (For our gedanken experiment the maximum value of $\Delta_{d}$ is $0.24 \mathrm{~nm}$.) The formula for $\sigma_{d}$ contains three terms. The first term, $1 / 2$, corresponds to the quantum dispersion of the initial wave function. The second term, $D \beta \tau$, describes the initial diffusion of an ensemble of oscillators. Formally, setting $\tau \sim 1 / \beta$, we can estimate the final dispersion $\sigma_{d}=\sqrt{D}$, which corresponds to thermodynamical vibrations of the cantilever tip. The third term describes insignificant oscillations with small amplitude, $D \beta$.

Note that the condition for distinguishing two cantilever positions at the beginning of the transient process is much less restrictive than the corresponding condition for the equilibrium positions at $\beta \tau>>1$. Indeed, after the first half-period $(\tau=\pi)$, we have $\Delta_{d}=4 \eta$ and $\sigma_{d}=(1 / 2+\pi D \beta)^{1 / 2}$. Taking into account that $\beta \ll 1$, the condition for distinguishing two positions, $\eta>(1 / 2+\pi D \beta)^{1 / 2}$, is much easier than $\eta>\sqrt{D}$. In our gedanken experiment the condition for distinguishing the two positions for the transient process is

$$
T<T_{\text {max }}=\frac{4}{\pi} \frac{Q F^{2}}{k_{B} k_{c}}=14 K,
$$

compared with $T<T_{\max }=1.7 \mathrm{mK}$ for the static Stern-Gerlach effect. This estimate seems to be too optimistic. It is connected with the very small distance $(5 \mathrm{~nm})$ between the ferromagnetic particle and the paramagnetic atom. If we increase this distance to $50 \mathrm{~nm}$, the temperature $T_{\max }$ drops from $14 \mathrm{~K}$ to $1.1 \mathrm{mK}$. Note that expression (39) coincides exactly with our preliminary estimates (2).

The condition $\Delta_{d}>2 \sigma_{d}$ is satisfied for the first time at

$$
\tau=\tau_{0} \approx 2^{1 / 4} / \sqrt{\eta}
$$

This expression is valid if $\eta>>1$ and $\eta>>(D \beta)^{2} / \sqrt{8}$. For our gedanken experiment we have $\eta=144, D=1.25 \times 10^{7} \mathrm{~T}$ ( $T$ is the temperature in Kelvin), $\beta=1.5 \times 10^{-4}$, and $\tau_{0}=0.1$. Thus, the above conditions are both satisfied. The value of $t_{0}=\omega_{c} \tau_{0}$ is approximately $9.3 \mu \mathrm{s}$. 


\section{Solution for off-diagonal spin matrix ele- ments}

The equations for the characteristics are now given by

$$
d \tau=\frac{d r}{\beta r-k}=\frac{d k}{r-4 \eta s}=\frac{d \hat{\rho}_{s,-s}}{-D \beta r^{2} \hat{\rho}_{s,-s}},
$$

or

$$
\begin{array}{ll}
\frac{d r}{d \tau} & =\beta r-k \\
\frac{d k}{d \tau} & =r-4 \eta s, \\
\frac{d \hat{\rho}_{s,-s}}{d \tau} & =-D \beta r^{2} \hat{\rho}_{s,-s} .
\end{array}
$$

The solutions of the first two equations of (42) are

$$
\begin{aligned}
& k=e^{\beta \tau / 2}\left(c_{1} \cos \theta \tau+c_{2} \sin \theta \tau\right)+4 \beta \eta s \\
& r=e^{\beta \tau / 2}\left[\left(\frac{\beta}{2} \cos \theta \tau-\theta \sin \theta \tau\right) c_{1}+\left(\frac{\beta}{2} \sin \theta \tau+\theta \cos \theta \tau\right) c_{2}\right]+4 \eta s .
\end{aligned}
$$

Following the same steps as above we obtain for the Fourier transform:

$$
\begin{array}{r}
\hat{\rho}_{1 / 2,-1 / 2}(k, r, \tau) \propto A\left(c_{1}, c_{2}\right) \exp \left\{-D \beta\left[f_{1} c_{1}^{2}+2 c_{1} c_{2} f_{3}+f_{2} c_{2}^{2}\right]\right\} \\
\times \exp \left\{-D \beta\left[4 \eta\left(g_{1} c_{1}+g_{2} c_{2}\right)+4 \eta^{2} \tau\right]\right\}
\end{array}
$$

where we fix $s=1 / 2$ (changing sign of $s$ corresponds to a change of sign of $\eta$, see Eq.(42), therefore the case $s=-1 / 2$ can be easily obtained). The functions $f_{i}$ and $g_{i}$ have been defined as above, and $c_{1}$ and $c_{2}$ are new characteristic curves given by

$$
\begin{aligned}
& c_{1}=e^{-\beta \tau / 2}\left(q_{1} k+q_{2} r+\eta q_{3}\right), \\
& c_{2}=e^{-\beta \tau / 2}\left(p_{1} k+p_{2} r+\eta p_{3}\right) .
\end{aligned}
$$


Here, $q_{1}, q_{2}, p_{1}, p_{2}$ are defined by Eqs. (22) and $q_{3}, p_{3}$ are given by

$$
\begin{aligned}
& q_{3}=\frac{2}{\theta}\left[-\beta\left(\frac{\beta}{2} \sin \theta \tau+\theta \cos \theta \tau\right)+\sin \theta \tau\right], \\
& p_{3}=\frac{2}{\theta}\left[\beta\left(\frac{\beta}{2} \cos \theta \tau-\theta \sin \theta \tau\right)-\cos \theta \tau\right] .
\end{aligned}
$$

With the same initial condition Eq. (15), we can determine the function $A\left(c_{1}, c_{2}\right)$ and obtain

$$
\begin{aligned}
& \hat{\rho}_{1 / 2,-1 / 2}(k, r, \tau) \propto \hat{\rho}_{1 / 2,-1 / 2}\left(c_{1}+2 \beta \eta, \frac{1}{2} \beta c_{1}+\theta c_{2}+2 \eta, 0\right) \\
& \quad \times \exp \left\{-D \beta\left[F_{1} c_{1}^{2}+2 c_{1} c_{2} F_{3}+F_{2} c_{2}^{2}+4 \eta\left(G_{1} c_{1}+G_{2} c_{2}\right)+4 \eta^{2} \tau\right]\right\},
\end{aligned}
$$

where $F_{i}(\tau)$ and $G_{i}(\tau)$ are defined as above. By substituting the initial condition (15) we have

$$
\begin{aligned}
\hat{\rho}_{1 / 2,-1 / 2}(k, r, \tau) \propto \exp \left\{i\left[\left(\frac{1}{2} p_{0} \beta+z_{0}\right) c_{1}+p_{0} \theta c_{2}+2 \eta\left(p_{0}+z_{0} \beta\right)\right]\right\} \\
\times \exp \left\{-\left[\left(\frac{\beta^{2}}{16}+\frac{1}{4}\right) c_{1}^{2}+\frac{\beta \theta}{4} c_{1} c_{2}+\frac{\theta^{2} c_{2}^{2}}{4}\right]\right\} \\
\times \exp \left\{-\left\{4 \eta\left[\left(\frac{3 \beta}{8}\right) c_{1}+\frac{\theta}{4} c_{2}\right]+\eta^{2}\left(1+\beta^{2}\right)\right\}\right\} \\
\times \exp \left\{-D \beta\left[F_{1} c_{1}^{2}+2 c_{1} c_{2} F_{3}+F_{2} c_{2}^{2}+4 \eta s\left(G_{1} c_{1}+G_{2} c_{2}\right)+\eta^{2} \tau\right]\right\},
\end{aligned}
$$

which can be written as

$$
\begin{aligned}
\hat{\rho}_{1 / 2,-1 / 2}(k, r, \tau) \propto & \exp \left[-r^{2} C_{12}-r \eta C_{11}-\eta^{2} C_{10}+i r C_{21}+i \eta C_{20}\right] \\
& \times \exp \left[\left(i B_{20}-r B_{11}-\eta B_{10}\right) k-\sigma_{*}^{2} k^{2}\right],
\end{aligned}
$$

where $\sigma_{*}$ is given by (29) and 


$$
\begin{aligned}
& C_{12}=e^{-\beta \tau}\left[\left(\frac{\beta^{2}}{16}+\frac{1}{4}+D \beta F_{1}\right) q_{2}^{2}+\left(\frac{\beta \theta}{4}+2 D \beta F_{3}\right) q_{2} p_{2}+\left(\frac{\theta^{2}}{4}+D \beta F_{2}\right) p_{2}^{2}\right], \\
& C_{11}=e^{-\beta \tau}\left[\left(\frac{\beta^{2}}{16}+\frac{1}{4}+D \beta F_{1}\right) 2 q_{2} q_{3}+\left(\frac{\beta \theta}{4}+2 D \beta F_{3}\right)\left(q_{2} p_{3}+p_{2} q_{3}\right)\right] \\
& +e^{-\beta \tau}\left[\left(\frac{\theta^{2}}{4}+D \beta F_{2}\right) 2 p_{2} p_{3}\right] \\
& +4 e^{-\beta \tau / 2}\left[\left(\frac{3 \beta}{8}+D \beta G_{1}\right) q_{2}+\left(\frac{\theta}{4}+D \beta G_{2}\right) p_{2}\right] \text {, } \\
& C_{10}=e^{-\beta \tau}\left[\left(\frac{\beta^{2}}{16}+\frac{1}{4}+D \beta F_{1}\right) q_{3}^{2}+\left(\frac{\beta \theta}{4}+2 D \beta F_{3}\right) q_{3} p_{3}+\left(\frac{\theta^{2}}{4}+D \beta F_{2}\right) p_{3}^{2}\right] \\
& +4 e^{-\beta \tau / 2}\left[\left(\frac{3 \beta}{8}+D \beta G_{1}\right) q_{3}+\left(\frac{\theta}{4}+D \beta G_{2}\right) p_{3}\right] \\
& +4\left(\frac{1}{4}+\frac{\beta^{2}}{4}+D \beta \tau\right) \text {, } \\
& C_{21}=e^{-\beta \tau / 2}\left[\left(\frac{p_{0} \beta}{2}+z_{0}\right) q_{2}+p_{0} \theta p_{2}\right] \\
& C_{20}=e^{-\beta \tau / 2}\left[\left(\frac{p_{0} \beta}{2}+z_{0}\right) q_{3}+p_{0} \theta p_{3}\right]+2\left(p_{0}+z_{0} \beta\right), \\
& B_{11}=e^{-\beta \tau}\left[\left(\frac{\beta^{2}}{16}+\frac{1}{4}+D \beta F_{1}\right) 2 q_{2} q_{1}+\left(\frac{\beta \theta}{4}+2 D \beta F_{3}\right)\left(q_{1} p_{2}+q_{2} p_{1}\right)\right] \\
& +e^{-\beta \tau}\left[\left(\frac{\theta^{2}}{4}+D \beta F_{2}\right) 2 p_{2} p_{1}\right] \\
& B_{10}=e^{-\beta \tau}\left[\left(\frac{\beta^{2}}{16}+\frac{1}{4}+D \beta F_{1}\right) 2 q_{3} q_{1}+\left(\frac{\beta \theta}{4}+2 D \beta F_{3}\right)\left(q_{1} p_{3}+q_{3} p_{1}\right)\right] \\
& +e^{-\beta \tau}\left[\left(\frac{\theta^{2}}{4}+D \beta F_{2}\right) 2 p_{3} p_{1}\right] \\
& +4 e^{-\beta \tau / 2}\left[\left(\frac{3 \beta}{8}+D \beta G_{1}\right) q_{1}+\left(\frac{\theta}{4}+D \beta G_{2}\right) p_{1}\right] \text {, } \\
& B_{20}=e^{-\beta \tau / 2}\left[\left(\frac{1}{2} p_{0} \beta+z_{0}\right) q_{1}+p_{0} \theta p_{1}\right]
\end{aligned}
$$

Performing the inverse Fourier transform we obtain 


$$
\begin{aligned}
\rho_{1 / 2,-1 / 2}(R, r, \tau)= & \frac{a b^{*}}{\sqrt{\pi} \sigma_{*}} \exp \left[-r^{2} C_{12}-r \eta C_{11}-\eta^{2} C_{10}+i r C_{21}+i \eta C_{20}\right] \\
& \times \exp \left[\left(-r B_{11}-\eta B_{10}+i B_{20}-i R\right)^{2} / 4 \sigma_{*}^{2}\right], \\
\rho_{-1 / 2,1 / 2}(R, r, \tau)= & \frac{a^{*} b}{\sqrt{\pi} \sigma_{*}} \exp \left[-r^{2} C_{12}+r \eta C_{11}-\eta^{2} C_{10}+i r C_{21}-i \eta C_{20}\right] \\
& \times \exp \left[\left(-r B_{11}+\eta B_{10}+i B_{20}-i R\right)^{2} / 4 \sigma_{*}^{2}\right] .
\end{aligned}
$$

Taking the modulus one obtains

$$
\begin{aligned}
& \left|\rho_{1 / 2,-1 / 2}(R, r, \tau)\right|=\frac{\left|a b^{*}\right|}{\sqrt{\pi} \sigma_{*}} e^{\xi \eta^{2}} e^{-\left(r+r_{0} \eta\right)^{2} / 2 \tilde{\sigma}^{2}} e^{-\left(B_{20}-R\right)^{2} / 4 \sigma_{*}^{2}} \\
& \left|\rho_{-1 / 2,1 / 2}(R, r, \tau)\right|=\frac{\left|a^{*} b\right|}{\sqrt{\pi} \sigma_{*}} e^{\xi \eta^{2}} e^{-\left(r-r_{0} \eta\right)^{2} / 2 \tilde{\sigma}^{2}} e^{-\left(B_{20}-R\right)^{2} / 4 \sigma_{*}^{2}}
\end{aligned}
$$

where

$$
\begin{aligned}
\tilde{\sigma}^{2} & =\frac{2 \sigma_{*}^{2}}{4 \sigma_{*}^{2} C_{12}-B_{11}^{2}}, \\
r_{0} & =\frac{2 \sigma_{*}^{2} C_{11}-B_{11} B_{10}}{4 \sigma_{*}^{2} C_{12}-B_{11}^{2}}, \\
\xi & =\frac{B_{10}^{2}}{4 \sigma_{*}^{2}}-C_{10}-\frac{r_{0}^{2}}{2 \tilde{\sigma}^{2}} .
\end{aligned}
$$

The maxima are located at $\left(R=B_{20}, r=-\eta r_{0}\right)$ for $\left.\left|\rho_{1 / 2,-1 / 2}\right|\right)$ and at $\left(R=B_{20}, r=\eta r_{0}\right)$ for $\left|\rho_{-1 / 2,1 / 2}\right|$. In $\left(z, z^{\prime}\right)$ coordinates this corresponds to $M_{+-}=\left(z=B_{20}-\eta r_{0} / 2, z^{\prime}=B_{20}+\eta r_{0} / 2\right)$ for $\left|\rho_{1 / 2,-1 / 2}\right|$ and $M_{-+}=$ $\left(z=B_{20}+\eta r_{0} / 2, z^{\prime}=B_{20}-\eta r_{0} / 2\right)$ for $\left|\rho_{-1 / 2,+1 / 2}\right|$, so that the distance between them is given by $\Delta_{n d}=\sqrt{2} \eta\left|r_{0}\right|$. Next, we consider the quadratic form $\left(r \pm r_{0} \eta\right) / 2 \tilde{\sigma}^{2}+\left(B_{20}-R\right)^{2} / 4 \sigma_{*}^{2}$ in the $\left(z, z^{\prime}\right)$ plane. Straightforward calculations show that this is an ellipse whose semi-axes are respectively given by $\tilde{\sigma}$ (across the diagonal) and $2 \sqrt{2} \sigma_{*}$ (along the diagonal). The centers of the peaks $M_{+-}, M_{-+}$are symmetric with respect to the diagonal line $z=z^{\prime}$.

The most remarkable difference compared with the diagonal case, is the 
presence of irreversible decoherence. Indeed, the heights of the peaks are exponentially reduced in time by the damping factor $\sim \exp \left(-4 \eta^{2} D \beta \tau\right)$. This, in turn, defines a characteristic time scale of decoherence: $\tau_{d}=1 / 4 \eta^{2} D \beta$. This formula exactly coincides with the expression derived in [1], based on a semi-qualitative analysis. At time $\tau=\tau_{0}$, when two diagonal peaks are clearly separated, the damping factor is $4 \eta^{2} D \beta \tau_{0}$. We expect to observe the coherence between the two peaks (MSCS) if this factor is not much more than one unit. Thus, using the expression for $\tau_{0}$ from Eq. (40), we can estimate the condition for the quantum coherence as $D \beta \eta^{3 / 2}<1$, or

$$
T<T_{\max }=\frac{Q}{k_{B}} \frac{\left(\hbar \omega_{c}\right)^{7 / 4} k_{c}^{3 / 4}}{F^{3 / 2}} .
$$

For our gedanken experiment the value of $T_{\max }$ is approximately $3 \times 10^{-7} \mathrm{~K}$.

Now, we will check the validity of our estimate for the parameters chosen for our gedanken experiment. Our solution is valid if $\eta>>(D \beta)^{2} / \sqrt{8}$. Setting $T=T_{\max }$ or $D \beta \eta^{3 / 2}=1$, we obtain $\eta^{4}>>1 / \sqrt{8}$, which is definitely true, assuming $\eta>>1$. Next, the condition of the validity of the high temperature approximation is $D>>1$. For $T=T_{\max }$, it follows that $\eta^{3 / 2}<<Q$. This inequality is roughly satisfied $\left(\eta^{3 / 2}=1700, Q=6700\right)$. Finally, as we mentioned in the Introduction, the master equation fails at times $t \leq \hbar / k_{B} T$. Thus, the time considered, $\tau_{0}=2^{1 / 4} / \sqrt{\eta}$, must be much greater than $1 / D$, which is definitely wrong. Thus, our condition (54) for the creation of MSCS is not justified for the parameters considered.

Next, we discuss at what values of the parameters a MSCS can be generated. First, we emphasize the qualitative difference between two conditions. 1) The condition for distinguishing two positions of the cantilever. 2) The condition for distinguishing two position of the cantilever and the coherence between these two positions. The first condition is relatively simple: $T<T_{\max }=F^{2} / k_{B} k_{c}$ for equilibrium positions $(\tau>>Q)$ and $T<T_{\max }=4 Q F^{2} / \pi k_{B} k_{c}$ for $\tau=\pi$. The obvious way to increase $T_{\max }$ is by decreasing the spring constant $k_{c}$ or increasing the magneto-static force $F$. For $\tau=\pi$, one additional way is to increase the quality factor $Q$. Condition 2) for generating an MSCS can be satisfied at temperature $T<T_{\max }=\hbar^{7 / 4} Q k_{c}^{13 / 8} / k_{B} F^{3 / 2} m^{7 / 8}$, where $m$ is the effective mass of the 
cantilever, $m=k_{c} / \omega_{c}^{2}$. At $T=T_{\max }$, the MSCS will be generated if we satisfy the inequalities $1 \ll \eta^{2} \ll Q$, or

$$
1<<\frac{m F^{2}}{\hbar k_{c}^{3 / 2}} \ll Q .
$$

One can see that the regime considered in our paper does not allow free manipulation of any parameter but $Q$. Increasing $Q$, we can satisfy the second inequality and, at the same time, increase $T_{\max }$. In our gedanken experiment, the tenfold increase of $Q(Q=67000)$ provides the validity of the right-hand inequality in (55) and increases $T_{\max }$ to $3 \mu \mathrm{K}$.

\section{Conclusion}

We have shown that the maximum temperature for a single spin measurement in MFM can be increased by a factor of $Q$ if one utilizes the initial transient process instead of the static displacement of the cantilever tip. We have obtained an exact analytical solution of the master equation, which describes the $Q$-times magnification of the maximum temperature. In addition, we have found the conditions for generation of macroscopic Schrödinger cat state in MFM.

\section{Acknowledgments}

The work of GPB was supported by the Department of Energy (DOE) under the contract W-7405-ENG-36. GPB and VIT thank the National Security Agency (NSA), the Advanced Research and Development Activity (ARDA), and DARPA (MOSAIC) for partial support. 


\section{References}

[1] G.P. Berman, G.D. Doolen, P.C. Hammel, and V.I.Tsifrinovich, Phys. Rev. A, 6503 (2002) 032311.

[2] A.O. Caldeira and A.T. Leggett, Physica A, 121 (1983) 587;

Ann. Phys. (N.Y.) 149 (1983) 374.

[3] W.G. Unruh and W.H. Zurek, Phys. Rev. D, 40 (1989) 1071.

[4] A. Venugopalan, Phys. Rev. A, 56 (1997) 4307.

[5] B.L. Hu, J.P. Paz, and Y. Zhang, Phys. Rev. D, 45 (1992) 2843.

[6] T.D. Stowe, K. Yasumura, T.W. Kenny, D. Botkin, K. Wago, and D. Rugar, Appl. Phys. Lett., 71 (1997) 288. 


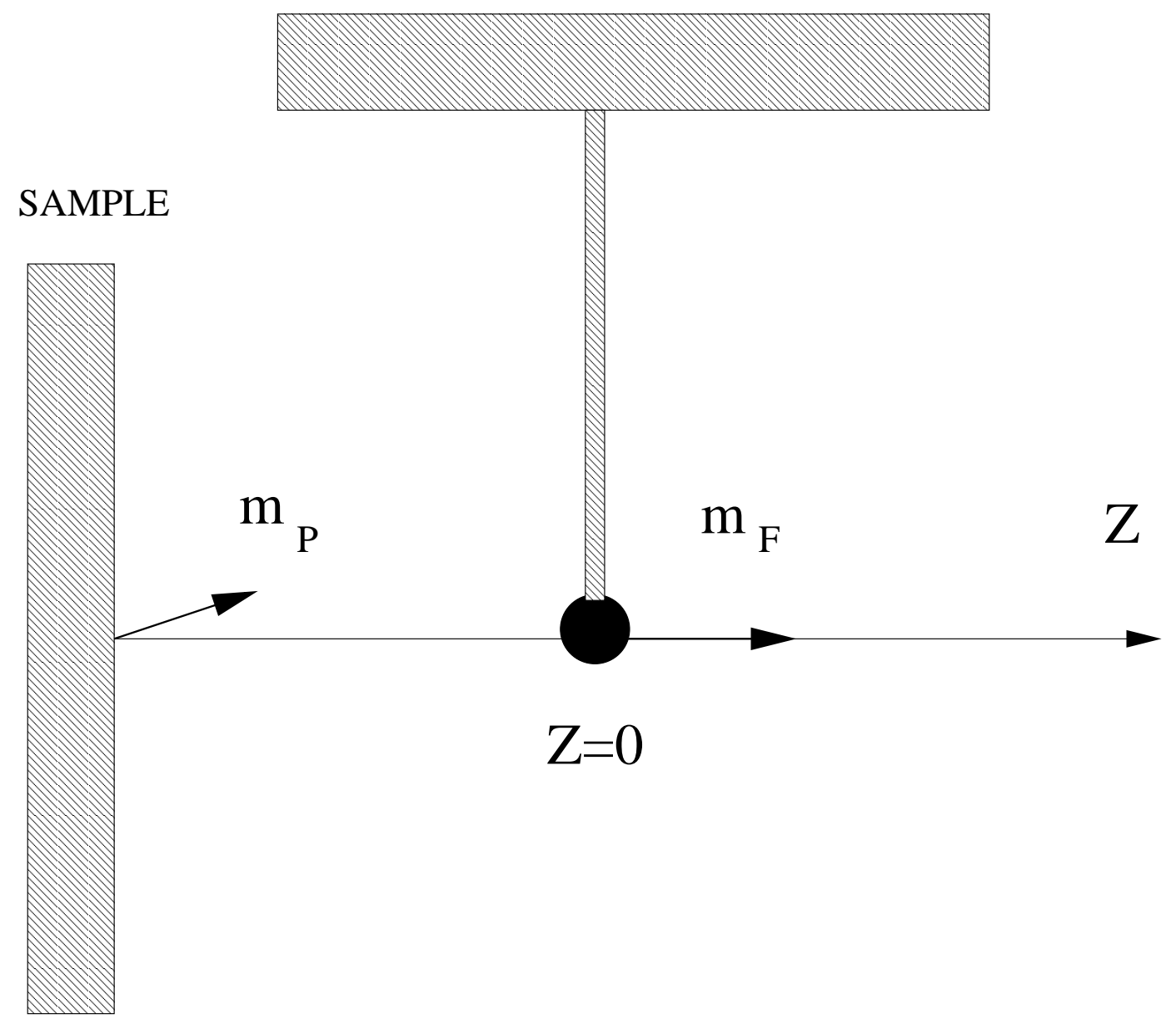

Figure 1: Geometry of the proposed gedanken experiment. $\mathbf{m}_{F}$ and $\mathbf{m}_{p}$ are the magnetic moments of the ferromagnetic particle and paramagnetic atom. 


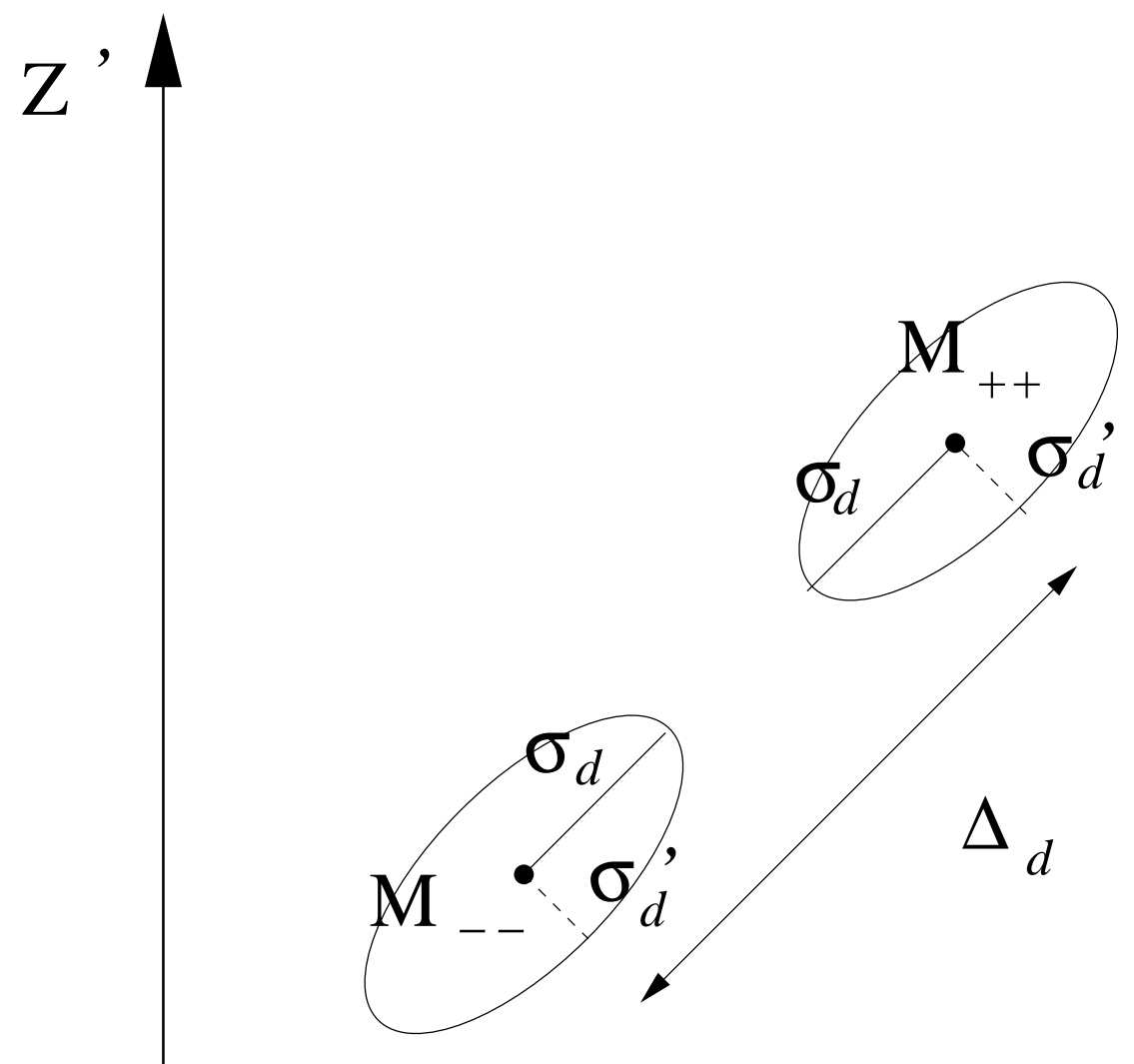

Z

Figure 2: Schematic view of the Gaussians representing the diagonal elements $\left|\rho_{-1 / 2,-1 / 2}\right|$ and $\left|\rho_{1 / 2,1 / 2}\right|$ (seen from the top) in the $\left(z, z^{\prime}\right)$ plane. We show the centers $M_{--}$and $M_{++}$, the variances $\sigma_{d}^{\prime}$ (transverse) and $\sigma_{d}$ (parallel), and the distance between diagonal centers $\Delta_{d}$. 